\title{
Actividades académico-asistenciales registradas por los egresados de la especialidad de Urgencias del Hospital Regional No. 25 del Instituto Mexicano del Seguro Social (1991-2018)
}

\author{
Academic-assistance activities registered by the graduates of the specialty of Emergency \\ of the Regional Hospital No. 25 of the Instituto Mexicano del Seguro Social (1991-2018)
}

Jorge Loría-Castellanos ${ }^{*}$, Juan C. Sánchez-Echeverria1, Felipe Cruz-Vega ${ }^{1}$, Erandy Montes de Oca-García ${ }^{2}$ y Álvaro J. Montiel-Jarquin ${ }^{3}$

'División de Proyectos Especiales en Salud, Dirección de Prestaciones Médicas, Instituto Mexicano del Seguro Social (IMSS), Ciudad de México; ${ }^{2}$ Hospital General 197, IMSS, Estado de México; ${ }^{3}$ Unidad Médica de Alta Especialidad, Hospital de Especialidades, IMSS, Puebla. México

\begin{abstract}
Resumen
Objetivo: Describir las actividades académico-asistenciales de los egresados de la especialidad de urgencias en el Hospital General Regional 25 del Instituto Mexicano del Seguro Social (IMSS). Método: Estudio observacional de tipo descriptivo en el que se analizaron las 25 generaciones egresadas de la especialidad. Se analizó la productividad científica, docente y asistencial de sus egresados, así como la formación posterior y los puestos directivos desempeñados. Resultados: De 1991 a 2018 ingresaron 238 residentes, de los cuales el 88.65\% concluyó la especialidad. El 57.34\% son de sexo masculino. El 78.67\% de los egresados laboran exclusivamente en el IMSS y el $68.72 \%$ lo hacen en más de una institución. El 72\% cuentan con certificación. Publicaron 112 artículos científicos y 4 libros. El 12.32\% de los egresados cursaron una segunda especialidad, siendo la predominante la de Atención del Paciente en Estado Crítico (84.61\%). Entre los logros principales de la sede están el de ser la primera sede de urgencias del país en ser incluida en el Programa de Posgrados de Calidad del Consejo Nacional de Ciencia y Tecnología. El 53\% de estos especialistas se desempeñan o han desempeñado en puestos directivos. Conclusiones: Esta sede se constituye como un referente dentro de la especialidad de urgencias en Latinoamérica.
\end{abstract}

Palabras Clave: Historia. Medicina de urgencia. Productividad. Programas de formación.

\begin{abstract}
Objective: To describe the academic-assistance activities of the graduates of the specialty of emergencies in the Regional General Hospital 25 of the Mexican Institute of Social Security. Method: Observational descriptive type study in which the 25 generations graduated from the specialty will be analyzed. The scientific, teaching and care productivity of its graduates was analyzed, as well as the subsequent training and the managerial positions held. Results: From 1991 to 2018238 residents entered, of which $88.65 \%$ concluded the specialty. $57.34 \%$ are male. $78.67 \%$ of the graduates work exclusively in the IMSS and $68.72 \%$ do it in more than one institution. $72 \%$ of them have certification. They published 112 scientific articles and 4
\end{abstract}

\footnotetext{
Correspondencia:

*Jorge Loría-Castellanos

Paseo de la Reforma, 476

Col. Juárez, Alcaldía Cuauhtémoc

Fecha de recepción: 12-12-2018

Cir Cir. 2020;88(2):137-142

C.P. 06600, Ciudad de México, México

Fecha de aceptación: 26-04-2019

Cir Cir. 2020;88(2):137-142

E-mail: jloriac@ hotmail.com

DOI: 10.24875/CIRU.19000962

www.cirugiaycirujanos.com

0009-7411/@ 2019 Academia Mexicana de Cirugía. Publicado por Permanyer. Este es un artículo open access bajo la licencia CC BY-NC-ND (http://creativecommons.org/licenses/by-nc-nd/4.0/).
} 
books. $12.32 \%$ of the graduates attended a second specialty, being the predecessor of patient care in the critical state (84.61\%). Among the main achievements of the headquarters are in the first emergency room of the country in the Quality Postgraduate Program of Consejo Nacional de Ciencia y Tecnología. 53\% of these specialists work or perform in management positions. Conclusions: This office is considered a reference in the specialty of emergencies in Latin America.

Key Words: History. Emergency medicine. Productivity. Training programs.

\section{Introducción}

La Federación Internacional de Medicina de Emergencia establece a esta como una especialidad médica que se basa en el conocimiento y las destrezas requeridas con la principal misión de prevenir, diagnosticar y tratar los aspectos agudos y urgentes de la enfermedad o de las lesiones que afectan a pacientes de todos los grupos de edad, con diferentes enfermedades no diferenciadas y de conducta. Además, abarca la responsabilidad y el desarrollo de sistemas médicos hospitalarios y prehospitalarios, creando las herramientas necesarias para su crecimiento ${ }^{1,2}$.

El médico especialista en medicina de urgencias es el pilar de su servicio, dirigiéndolo y estableciendo los estándares de calidad que se requieren basándose en la jerarquización de los problemas más graves de salud $^{3-5}$.

En México, la especialidad de medicina de urgencias surge como una necesidad de personal capacitado para la atención inicial del paciente agudo en condiciones críticas. Esto se hizo evidente tanto la explosión de San Juanico en 1984 como en el terremoto de 1985 en la Ciudad de México, en donde hubo falta de organización y de capacitación de los sistemas de salud en la atención de pacientes en casos de desastre ${ }^{6,7}$.

A partir de 1986, el Hospital General Balbuena, de los entonces Servicios Médicos del Departamento del Distrito Federal, estableció la especialidad de Urgencias Médico Quirúrgicas con el objeto de preparar médicos capacitados para la atención de pacientes en estado crítico en el servicio de urgencias ${ }^{3}$.

Dentro del Instituto Mexicano del Seguro Social (IMSS), la especialidad se inicia 5 años después, en 15 sedes en todo el país. Una de estas sedes fue el Hospital General Regional 25 («clínica 25»), ubicado en la Calzada Ignacio Zaragoza de la Delegación Iztapalapa del Distrito Federal, contando desde su origen con el aval académico de la Escuela Superior de Medicina del Instituto Politécnico Nacional ${ }^{8}$.

El Hospital General Regional No. 25 del IMSS contó en su momento con el servicio de urgencias más grande de Latinoamérica. De forma habitual, sus 37 camas se veían con mucho saturadas y los pacientes hospitalizados en ellas superaban los 150. Contaba con una unidad de reanimación en la que se estabilizaba un promedio de 15 pacientes por turno, además de realizar un sinfín de procedimientos. Contaba además con un área de urgencias de pediatría, con 30 camas. Su plantilla la conformaban 76 médicos, en su mayoría urgenciólogos, pero que se complementaban con otras especialidades (cirugía, pediatría, medicina interna, etc.) para atender un promedio de 450 consultas diarias ${ }^{9}$.

Derivado de los sismos del 7 y el 19 de septiembre de 2017, el Hospital General Regional No. 25 sufrió daños estructurales importantes. Posterior a los tres peritajes a que se sometió, se concluyó que era necesario reubicar sus servicios médicos en cumplimiento de las disposiciones en materia de protección civil para garantizar la seguridad de los derechohabientes, de los médicos y de todo el personal que ahí labora.

Aunque se instrumentó un plan de acción para garantizar la continuidad en la atención de los servicios de urgencia y de las consultas programadas de especialidades en unidades aledañas, es una realidad que la funcionalidad de la residencia médica se verá afectada al carecer no solo de la infraestructura habitual, sino también de la plantilla docente y asistencial que a lo largo del tiempo ha fortalecido la sede ${ }^{10}$.

En este sentido, consideramos un buen momento para hacer un recuento de los logros de la especialidad emblemática de este hospital: urgencias médico-quirúrgicas.

\section{Método}

Estudio observacional de tipo transversal descriptivo en el que se realizó el seguimiento de todos los egresados de la especialidad de urgencias del Hospital General Regional 25, del IMSS, desde 1991 hasta 2018.

Se analizaron por generación la eficiencia terminal, la productividad científica (presentaciones en congresos, artículos y libros publicados), los reconocimientos recibidos, los logros obtenidos, la formación académica posterior a la especialidad de urgencias y 
los puestos directivos desempeñados. Se empleó estadística descriptiva.

\section{Resultados}

De 1991 a 2018 ingresaron al Hospital General Regional 25 del IMSS 238 residentes de la especialidad de urgencias, de los cuales 211 (88.65\%) la concluyeron. El $57.34 \%$ eran de sexo masculino. Las generaciones con más egresados tuvieron 14 , y la de menos tuvo 3. Desde el año 2011 han predominado las egresadas de sexo femenino (Tabla 1).

El $72 \%$ de los egresados cuentan con certificación por el Consejo Mexicano de Medicina de Urgencias.

Aunque todos los Estados del país cuentan con al menos un egresado de la especialidad de urgencias, el $54.97 \%$ laboran actualmente en el área metropolitana (Ciudad y Estado de México).

El $78.67 \%$ de los egresados laboran exclusivamente en el propio IMSS, en tanto que el $68.72 \%$ lo hacen en más de una institución (Tabla 2).

Los egresados de la especialidad han publicado un total de 112 artículos científicos. La generación de 1999 ha sido la más productiva (79.46\%), siendo los temas principales la investigación educativa (21.4\%) y los desastres (14.28\%) (Tablas 1 y 3 ).

Han publicado un total de 4 libros: $2(50 \%)$ sobre diagnóstico y manejo de urgencias, 1 (25\%) referente a historia de la medicina de urgencia en México y $1(25 \%)$ sobre toxicología clínica ${ }^{8,11-13}$.

Entre los principales reconocimientos obtenidos por estos egresados se encuentran:

- Diecinueve reconocimientos a la atención de calidad, IMSS.

- Nueve reconocimientos a la meritoria productividad científica, IMSS.

- Un Premio al Mérito Médico, en la categoría de Educación en Salud, Fundación IMSS-Afore XXI.

- Segundo lugar en el Premio «Académico Doctor Francisco Fonseca García», Academia Mexicana de Cirugía.

Han presentado 67 trabajos en congresos nacionales y 14 en congresos internacionales.

Entre sus principales logros se encuentran:

- Primera sede de urgencias del país en ser incluida en el Programa de Posgrados de Calidad del Consejo Nacional de Ciencia y Tecnología (CONACYT), con el nivel de Consolidado (2012).

- Primer urgenciólogo en ingresar a la Academia Mexicana de Cirugía: Académico Dr. Jorge Loría Castellanos (2012).
Tabla 1. Distribución por año de egreso, sexo y artículos publicados

\begin{tabular}{|c|c|c|c|c|}
\hline Año de egreso & Hombres & Mujeres & Total & Artículos publicados \\
\hline 1994 & 4 & 3 & 7 & 0 \\
\hline 1995 & 4 & 0 & 4 & 0 \\
\hline 1996 & 4 & 0 & 4 & 0 \\
\hline 1997 & 3 & 0 & 3 & 1 \\
\hline 1998 & 6 & 0 & 6 & 0 \\
\hline 1999 & 2 & 3 & 5 & 89 \\
\hline 2000 & 7 & 1 & 8 & 2 \\
\hline 2001 & 6 & 2 & 8 & 11 \\
\hline 2002 & 4 & 3 & 7 & 2 \\
\hline 2003 & 7 & 4 & 11 & 4 \\
\hline 2004 & 7 & 5 & 12 & 1 \\
\hline 2005 & 3 & 3 & 6 & 1 \\
\hline 2006 & 8 & 0 & 8 & 0 \\
\hline 2007 & 9 & 3 & 12 & 0 \\
\hline 2008 & 8 & 1 & 9 & 0 \\
\hline 2009 & 8 & 3 & 11 & 0 \\
\hline 2010 & 6 & 8 & 14 & 0 \\
\hline 2011 & 3 & 11 & 14 & 0 \\
\hline 2012 & 4 & 5 & 9 & 0 \\
\hline 2013 & 3 & 7 & 10 & 0 \\
\hline 2014 & 3 & 6 & 9 & 0 \\
\hline 2015 & 4 & 8 & 12 & 0 \\
\hline 2016 & 4 & 7 & 11 & 0 \\
\hline 2017 & 4 & 7 & 11 & 0 \\
\hline 2018 & 121 & 90 & 211 & 112 \\
\hline
\end{tabular}

- Primer presidente del Consejo Mexicano de Medicina de Urgencias no egresado de los Hospitales del Gobierno del Distrito Federal: Dra. Virginia Velasco Díaz.

- Primer presidente de la Sociedad Mexicana de Medicina de Emergencias no egresado de los Hospitales del Gobierno del Distrito Federal: Dr. Rubén Gutiérrez Luna.

- Participación como sinodales en todas las versiones del examen del Consejo.

- Coordinación nacional de los protocolos CRASH, CRASH 2 y CRASH 3, bajo auspicio de la London School of Hygiene and Tropical Medicine/ 
Tabla 2. Situación laboral de los egresados de la especialidad de urgencias, Hospital General Regional 25 (1991-2018)

\begin{tabular}{lcc}
\hline Institución laboral & $\mathbf{n}$ & $\%$ \\
\hline Fallecieron & 2 & 0.94 \\
No laboran & 4 & 1.89 \\
Instituto Mexicano del Seguro Social & 166 & 78.67 \\
Instituto de Seguridad y Servicios Sociales de los & 13 & 6.16 \\
Trabajadores del Estado & & \\
Secretaría de salud & 6 & 2.84 \\
Práctica privada & 11 & 5.21 \\
Universidades & 9 & 4.26 \\
Total & 211 & 100 \\
Más de una institución & 145 & 68.72 \\
\hline
\end{tabular}

Tabla 3. Temas de los artículos publicados por los egresados de la especialidad de urgencias, Hospital General Regional 25 (1991-2018)

\begin{tabular}{ll}
\hline Tema & $\mathbf{n}$ \\
\hline Investigación educativa & 24 \\
Desastres & 16 \\
Gerencia & 14 \\
Urgencias traumatológicas & 13 \\
Miscelánea & 12 \\
Toxicología & 9 \\
Urgencias de pediatría & 7 \\
Terapia intensiva & 7 \\
Procedimientos de urgencias & 5 \\
Urgencias neurológicas & 5 \\
Total & 112 \\
\hline
\end{tabular}

OPS, cuyos resultados se publicaron en The Lancet y British Medical Journal.

- Un candidato a Investigador Nacional dentro del Sistema Nacional de Investigadores del CONACYT.

Dos de sus egresados cuentan con calificación institucional como investigadores y siete con calificación docente.

Han cursado 71 diferentes diplomados, de los cuales predominan los de toxicología (30.98\%) (Tabla 4).

Veintiséis de los egresados (12.32\%) cursaron una segunda especialidad, siendo la predominante la de Atención del Paciente en Estado Crítico (84.61\%) (Tabla 5).
Tabla 4. Diplomados cursados por los egresados de la especialidad de urgencias, Hospital General Regional 25 (1991-2018)

\begin{tabular}{lc}
\hline Diplomado & $\mathbf{n}$ \\
\hline Toxicología & 22 \\
Docencia & 12 \\
Desastres & 12 \\
Ultrasonido & 11 \\
Investigación & 9 \\
Ética & 3 \\
Donación de órganos & 2 \\
Total & 71 \\
\hline
\end{tabular}

Tabla 5. Segunda especialidad o subespecialidad cursadas por los egresados de la especialidad de urgencias, Hospital General Regional 25 (1991-2018)

\begin{tabular}{ll}
\hline Especialidad o subespecialidad & $\mathbf{n}$ \\
\hline Atención del paciente en estado crítico & 22 \\
Bariatría & 1 \\
Patología & 1 \\
Radiología & 1 \\
Reanimatología & 1 \\
Total & 26 \\
\hline
\end{tabular}

Tabla 6. Puestos directivos desempeñados por los egresados de la especialidad de urgencias, Hospital General Regional 25 (1991-2018)

\begin{tabular}{lc}
\hline Puesto & $\mathbf{n}$ \\
\hline Directivo nivel central & 1 \\
Director de hospital & 6 \\
Subdirector de hospital & 22 \\
Jefe de servicio & 42 \\
Patología & 1 \\
Radiología & 1 \\
Terapia intensiva & 5 \\
Urgencias & 30 \\
Educación & 5 \\
Total & 113 \\
\hline
\end{tabular}

El $17.53 \%$ de los egresados han cursado un posgrado, siendo el $86.48 \%$ Maestría en administración de hospitales, el $10.81 \%$ Maestría en educación y el $2.7 \%$ Doctorado en educación.

El $53 \%$ de estos especialistas se desempeñan o han desempeñado en puestos directivos, predominando el de jefe de urgencias (26.54\%) (Tabla 6). 
Conformaron los equipos médicos de emergencias del IMSS que participaron en diversas situaciones de desastres, entre ellas las misiones de ayuda en las inundaciones de Tabasco (2008), el terremoto de Haití (2009) y los sismos de Oaxaca y Ciudad de México (2017).

\section{Discussión}

Lo primero en llamar la atención es la ubicación de la sede, que le dio desde sus orígenes unas características especiales, ya que se encuentra en los límites de Iztapalapa y ciudad Nezahualcóyotl, dos de las zonas más pobladas y violentas del país, lo que hace que los pacientes que acuden a su servicio de urgencias no solo saturen de forma cotidiana el servicio, sino que traslapen padecimientos traumatológicos con agudización de enfermedades crónicas degenerativas ${ }^{14}$.

No es de sorprender que la mayoría de los especialistas laboren en el mismo instituto que los formó, dadas las facilidades contractuales que se les ofrecen al egreso. Lo que sí resulta interesante es el elevado porcentaje que se desempeña en más de una institución, situación que pudiera derivar en afectaciones tanto de la salud de los profesionales como en un impacto negativo en el propio sistema de salud ${ }^{15}$.

Llama la atención el porcentaje de certificación de sus egresados, el cual es mayor que el de otras sedes y en lo que seguramente influye que sus profesores y egresados de generaciones anteriores formen parte de dicho proceso.

Es importante la distribución de estos médicos en todo el país, ya que no solo impacta en la calidad de la atención médica, sino también en la difusión del concepto de la especialidad.

Es interesante la productividad en materia de artículos científicos y libros, que no tiene un referente de comparación con otra sede de urgencias y que no solo abarca los temas "clásicos de la especialidad", sino que también considera aspectos que influyen en su desarrollo, implementación y mejora, como serían la investigación educativa o la propia historia de la especialidad. Lo que sí hay que recalcar es que son tres las generaciones (1999, 2001 y 2003) que más han publicado, situación que pudiera explicarse por el hecho de que estos egresados son los que se formaron posteriormente en docencia e investigación, así como que se desempeñaron o desempeñan como profesores o jefes de educación de esta y otras sedes.

Los reconocimientos recibidos no se limitan únicamente al desempeño que día a día se realiza en los servicios de urgencia, sino que engloban actividades complementarias, como la docencia y la investigación, lo que nos habla de profesionistas integrales.

Es importante la representación de sus egresados en los principales organismos de la medicina y de la especialidad en México, lo cual no es una mera presencia, pues su trabajo en realidad repercutirá en el sistema de salud en pleno.

Un punto que hay que destacar es el haber sido la primera sede en ser incluida en el Programa de Posgrados de Calidad del CONACYT, el cual reconoce la calidad de la formación de los programas a través de un riguroso proceso de evaluación por pares académicos. Con este programa se fomenta la mejora continua y se asegura la calidad del posgrado nacional "para incrementar las capacidades científicas, humanísticas, tecnológicas y de innovación del país, que incorporen la generación y aplicación del conocimiento como un recurso para el desarrollo de la sociedad y la atención a sus necesidades, contribuyendo así a consolidar el crecimiento dinámico y un desarrollo más equitativo y sustentable del país»16.

A diferencia de otros países, en donde el desarrollo académico de la especialidad de urgencias se desarrolla dentro de los departamentos de medicina interna o cirugía, el programa de esta sede se imparte con autonomía académica primordialmente en los diferentes servicios de urgencias de segundo y tercer nivel de atención del IMSS, con un cuerpo docente-asistencial bastante robusto que incluye formación no solo en las áreas clínicas, sino también en metodología de la investigación e incluso en ética ${ }^{17}$.

Es una realidad que la especialidad en México nació de un desastre, por lo que es trascendental que sus egresados no solo no se alejan de esta necesidad, sino también que sean parte clave de la respuesta ante emergencias mayores y desastres, tanto nacionales como internacionales, como se ha evidenciado en su reciente participación tras los sismos de septiembre de 2018 en México ${ }^{18}$.

El 12 de octubre de 2018, a las 24:00 h, con la finalidad de demolerlo y reconstruirlo, el Hospital General Regional 25 dejó de laborar oficialmente, y con él una de las sedes de la especialidad de urgencias más 
trascendentales de Latinoamérica. Podemos decir con satisfacción que los egresados de la especialidad de urgencias han cumplido con creces con la visión de esta: «Ser la sede líder en Latinoamérica en la formación de especialistas para la prevención, detección, atención, docencia e investigación de procesos y patologías de urgencia, a través de un enfoque de superación personal y profesional continuo del equipo de salud».

Estamos seguros de que solo es cuestión de tiempo para que, una vez reconstruido el hospital, se retome con fuerza este liderazgo.

\section{Conflicto de intereses}

Los autores declaran que no existe conflicto de intereses.

\section{Responsabilidades éticas}

Protección de personas y animales. Los autores declaran que para esta investigación no se han realizado experimentos en seres humanos ni en animales.

Confidencialidad de los datos. Los autores declaran que han seguido los protocolos de su centro de trabajo sobre la publicación de datos de pacientes.

Derecho a la privacidad y consentimiento informado. Los autores declaran que en este artículo no aparecen datos de pacientes.

\section{Bibliografía}

1. American College of Emergency Physicians. Definition of emergency medicine. (Consultado el 10 de octubre de 2018.) Disponible en: https://www. acep.org/patient-care/policy-statements/definition-of-emergency-medicine

2. Castillo-de los Santos R. Medicina de urgencias: una especialidad nueva para el gremio médico militar, pero vital para el servicio de sanidad militar. Rev Sanid Milit Mex. 2017;71:290-9.

3. Peña Viveros R. Calidad en medicina de urgencia, una necesidad de nuestros días (Parte I). Rev Mex Med Urg. 2002;1:31-6.

4. Valladares-Aranda MA. La medicina de urgencias en los tiempos modernos. Arch Med Urg Mex. 2010;2:3-4.

5. Vázquez-Delgado IM. El médico de urgencias: líder de su propia existencia. Rev Mex Med Urg. 2002;1:24-5.

6. Rocha-Luna JM. Historia de la medicina de urgencia en México. Arch Med Urg Mex. 2009;1:4-11.

7. Loría-Castellanos J, Flores-Maciel L, Márquez-Ávila G, Valladares-Aranda MA. Frecuencia y factores asociados con el uso inadecuado de la consulta de urgencias de un hospital. Cir Cir. 2010;78:508-14.

8. Loría-Castellanos J. Medicina de urgencia en México. Tres décadas de logros y retos. Editorial Berit. Ciudad de México, México, 2017.

9. Sistema de información del Instituto Mexicano del Seguro Social. IMSS. (Consultado el 10 de octubre de 2018.) Disponible en: https://iieg.gob. $\mathrm{mx} /$ contenido/PoblacionVivienda/Salud/PresentaSIdelIMSS.pdf.

10. Hospital General Regional No. 25 "Zaragoza" reubicará sus servicios. (Consultado el 8 de octubre de 2018.) Disponible en: http://www.imss. gob.mx/prensa/archivo/201810/Aviso.

11. Cabrera-Rayo A. Puesta al día en medicina interna, temas de urgencias. Editorial Alfil. Ciudad de México, México, 2012.

12. Villatoro-Martínez A, Loría-Castellanos J. Manual de medicina de urgencias del adulto mayor. Editorial Alfil. Ciudad de México, México, 2015.

13. Sánchez-Villegas MC, Loría-Castellanos J. Toxicología clínica en urgencias. Editorial Alfil. Ciudad de México, México, 2016.

14. Instituto Politécnico Nacional (IPN), Escuela Superior de Medicina, México. Programa de estudios de posgrado. México: IPN; 2018.

15. Loría-Castellanos J, Rocha-Luna JM, Márquez-Ávila G. Patrón y calidad subjetiva de sueño en médicos residentes y su relación con la ansiedad y la depresión. Emergencias. 2010;22:33-9.

16. Programa Nacional de Posgrados de Calidad. (Consultado el 10 de septiembre de 2018.) Disponible en: https://www.conacyt.gob.mx/index. php/becas-y-posgrados/programa-nacional-de-posgrados-de-calidad.

17. Mallon WK, Valenzuela R, Salway RJ, Shoenberger JM, Swadron SP. La especialidad de medicina de urgencia en Chile: 20 años de historia. Rev Med Clin Condes. 2017;28:170-7.

18. Cruz-Vega F, Loría-Castellanos J, Sánchez-Echeverría JC, Sosa-Barragán R. Experiencia de un equipo médico de emergencia del IMSS durante el sismo en México. Rev Panam Salud Pública. 2018;42:e79. 